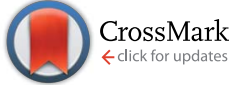

Cite this: RSC Adv., 2015, 5, 41786

Received 17th February 2015

Accepted 13th April 2015

DOI: $10.1039 / c 5 r a 03053 f$

www.rsc.org/advances

\section{Mediator-free total cholesterol estimation using a bi-enzyme functionalized nanostructured gold electrode $\uparrow$}

\begin{abstract}
Rachna Sharma, ${ }^{a b}$ R. K. Sinha ${ }^{b}$ and Ved Varun Agrawal ${ }^{\star a}$
We report the fabrication of a bi-enzyme functionalized nanostructured Au electrode for the mediator-free determination of total cholesterol. A one-step electrochemical route for the synthesis, functionalization and deposition of Au nanostructures via the electroreduction of gold chloride onto indium tin oxide (ITO) coated glass plates has been proposed. The covalent biofunctionalization of the optimized $\mathrm{Au}$ electrode was done with cholesterol esterase (ChEt) and cholesterol oxidase (ChOx) to investigate the kinetic parameters and the sensing characteristics. The $\mathrm{ChEt}-\mathrm{ChOx} / \mathrm{Glu}-\mathrm{NanoAu/ITO}$ bioelectrode has a surface-controlled electrode reaction with an electron transfer coefficient and a charge transfer rate constant of 0.68 and $7.09 \mathrm{~s}^{-1}$, respectively. Under the optimal conditions, the bioelectrode undergoes a direct electron transfer reaction and exhibits a high sensitivity of $0.53 \mathrm{~mA} \mathrm{mM} \mathrm{m} \mathrm{cm}^{-2}$ and a low detection limit of $1.57 \mu \mathrm{M}$ for cholesterol ester without the use of any redox mediator. In addition, the kinetic analysis reveals that the bioelectrode exhibits a surface concentration of $8.82 \times 10^{-12} \mathrm{~mol} \mathrm{~cm}{ }^{-2}$. The sensor has also been validated with clinical samples. The proposed biosensor shows good sensitivity, stability and selectivity towards total cholesterol and may thus find implications in the fabrication of biosensing devices.
\end{abstract}

\section{Introduction}

The unique electronic, optical and catalytic properties of gold nanoparticles (NPs) have inspired researchers to develop simpler methods for the synthesis of various nanostructures and intensified the study of their applications. ${ }^{1}$ The tunable surface chemistry, morphology, optical, catalytic and electrocatalytic properties of gold nanostructures make them ideal for a variety of sophisticated technology applications such as organic photovoltaics, ${ }^{2}$ optical sensing, ${ }^{3}$ drug delivery ${ }^{4}$ and other biomedical applications., ${ }^{5,6}$ Duncan et al. reported the tunability of the Au NP monolayer allowing for complete control of the surface properties for the targeting and release of drugs using these nanocarriers. ${ }^{7}$ The remarkable electrocatalytic activity, surface plasmon resonance and biocompatibility of nano-gold make it promising for applications in biochemical sensing and medical diagnostics. ${ }^{8}$ Si et al. reported the fabrication of a highly sensitive glucose biosensor based

${ }^{a}$ Biomedical Instrumentation Section, National Physical Laboratory, New Delhi 110012, India. E-mail: ved.varun@gmail.com; Tel: +91-11-45609489

${ }^{b}$ Department of Applied Physics, Delhi Technological University, New Delhi 110042, India

$\dagger$ Electronic supplementary information (ESI) available: Scheme showing changes in the cyclic voltammograms after each modification step onto ITO, cyclic voltammograms of the ChEt-ChOx/Glu-NanoAu/ITO bioelectrode in the presence of cholesterol and other interfering analytes. See DOI: 10.1039/c5ra03053f on covalently-assembled high density Au nanostructures. ${ }^{9}$ Although bulk gold is a poor catalyst, nanometer-sized gold exhibits excellent electrocatalytic activity and the electrochemical properties can be tuned by controlling the size and shape for the desired application. One important attribute of nanosized catalysts is their high surface area and their interface-dominated properties, which differ from their atomic, molecular and bulk counterparts. ${ }^{10} \mathrm{Au}$ nanostructures can provide a direct electrical communication between biomolecules and electrodes resulting in direct electron transfer across the interface. Shi et al. reported the fabrication of aloe-like gold micro/nanostructures for ultrasensitive DNA recognition. ${ }^{\mathbf{1 1}}$ Liu et al. have reported the direct electron transfer of copper, zincsuperoxide dismutase (Cu, Zn-SOD) onto nanospherical, nanorod-like, and nanopyramidal gold nanostructures resulting in a mediator-free superoxide anion biosensor. ${ }^{12}$

The integration of gold nanostructures into thin films is particularly important for multiple applications in electrochemistry and bioelectronics for the development of biomedical and optoelectronic nanodevices. Precise control over the arrangement of NPs on the substrate is of utmost importance. Several methods for preparing nanostructured Au films have been reported, however, most of them involve multiple steps for the synthesis, surface modification and assembly of the NPs. However, electrochemical techniques offer a rapid and uniform deposition of the desired materials and their composites by optimizing parameters such as solution concentration, applied 
potential, $\mathrm{pH}$ etc. ${ }^{\mathbf{1 3}}$ The electrodeposition of nanomaterials involves the use of hard templates such as anodic aluminium oxide and highly ordered pyrolytic graphite etc. Soft templates such as polymers or surfactants can also be used as shape controllers to direct the preferential growth of Au nanoparticles into desired shapes. Huang et al. reported the electrochemical tuning of the Au nanoparticle size using a tetradodecylammonium bromide surfactant. ${ }^{14}$ The template-free electrodeposition of $\mathrm{Au}$ nanostructures can be achieved using Au precursors like $\mathrm{HAuCl}_{4}$ and $\mathrm{Au}\left(\mathrm{PPh}_{3}\right) \mathrm{Cl}$. Wang et al. reported the fabrication of flower-like nanoparticles from $\mathrm{HAuCl}_{4}$ at $\mathrm{pH} 5.0$ using nanoplates as building blocks. ${ }^{15}$ Sharma et al. have reported the deposition of different $\mathrm{Au}$ nanostructures using $\mathrm{Au}\left(\mathrm{PPh}_{3}\right) \mathrm{Cl}$ by varying the concentration of the capping ligand. ${ }^{16}$

The efficient immobilization of biomolecules onto the nanostructured surface is crucial for the fabrication of biosensors. The desired functionalization of NPs can be accomplished during synthesis by the addition of a suitable capping ligand with terminations like $\mathrm{NH}_{2}, \mathrm{COOH}$ etc., which can be utilized for the covalent attachment of biomolecules. Biofunctionalized $\mathrm{Au}$ nanostructures enable a wide range of biomedical applications. For instance, Luo et al. demonstrated multiple applications of protein and peptide-protected Au nanocrystals (size $\sim 2$ $\mathrm{nm}$ ), such as optical probes for bioimaging and biosensing, and constructing antimicrobial and therapeutic agents. ${ }^{17}$ The utilization of cysteamine-functionalized nanostructured $\mathrm{Au}$ electrodes for the immobilization of enzyme molecules may improve the efficacy of biosensors due to the high loading of covalently-linked enzymes and the facilitation of direct electron transfer from the redox centres of biomolecules to the electrode. $^{16}$

In this manuscript, we report the deposition of $\mathrm{Au}$ nanostructures onto ITO electrodes using a chronoamperometric technique. The electrocatalytic activity of the nanostructured $\mathrm{Au}$ electrode has been optimized by varying the cysteamine concentration during the synthesis and the kinetic parameters of the Au electrodes were evaluated using cyclic voltammetry. The optimized Au electrode was utilized for the covalent immobilization of two enzymes (ChEt and ChOx) in order to investigate the interfacial kinetics of the bi- enzymatic electrode and its sensing performance towards total cholesterol.

\section{Materials and methods}

Cholesterol esterase, cholesterol oxidase, cholesterol oleate, tetrachloroauric acid $\left(\mathrm{HAuCl}_{4}\right)$, trichloroacetic acid, triton X-100 and cysteamine hydrochloride were purchased from SigmaAldrich. All reagents were of analytical grade and used without further purification. Deionized water (Milli Q 10 TS) with resistivity $>18.2 \mathrm{M} \Omega \mathrm{cm}$ was used for preparing all aqueous solutions. Indium-tin-oxide (ITO) coated glass plates were purchased from Balzers, UK, (Baltracom 247 ITO, $1.1 \mathrm{~mm}$ thick) with a sheet resistance and a transmittance of $25 \Omega \mathrm{sq}^{-1}$ and $90 \%$, respectively. The stock solution of cholesterol oleate was prepared in $10 \%$ triton $\mathrm{X}-100$ and stored at $4{ }^{\circ} \mathrm{C}$.

\subsection{Instrumentation}

Morphological studies were carried out using an Atomic Force Microscope (Nanoscope, Bruker) and a Scanning Electron Microscope (SEM, FEI Nova 600 microscope). Fourier Transform Infrared (FT-IR) studies were carried out on a Perkin Elmer, Spectrum BX II spectrophotometer. Contact angle measurements were carried out using a OCA 15 EC (DataPhysics). Electrochemical experiments were conducted on an Autolab PGSTAT 302N System (Ecochemie, The Netherlands) using a three electrode system with platinum as the auxiliary, $\mathrm{Ag} / \mathrm{AgCl}$ as the reference and the ITO substrate as the working electrode.

\subsection{Fabrication of the nanostructured Au electrode}

The ITO substrates were modified with different Au nanostructures using a chronoamperometric technique. An aqueous solution of $\mathrm{HAuCl}_{4}(0.10 \mathrm{mM})$ and trichloroacetic acid $(50 \mathrm{mM})$ was used as an electrolyte. For the fabrication of the nanostructured $\mathrm{Au}$ films, the deposition potential and deposition time were optimized as $-1 \mathrm{~V}$ and $300 \mathrm{~s}$, respectively. The morphology of the Au electrodes was controlled by varying the

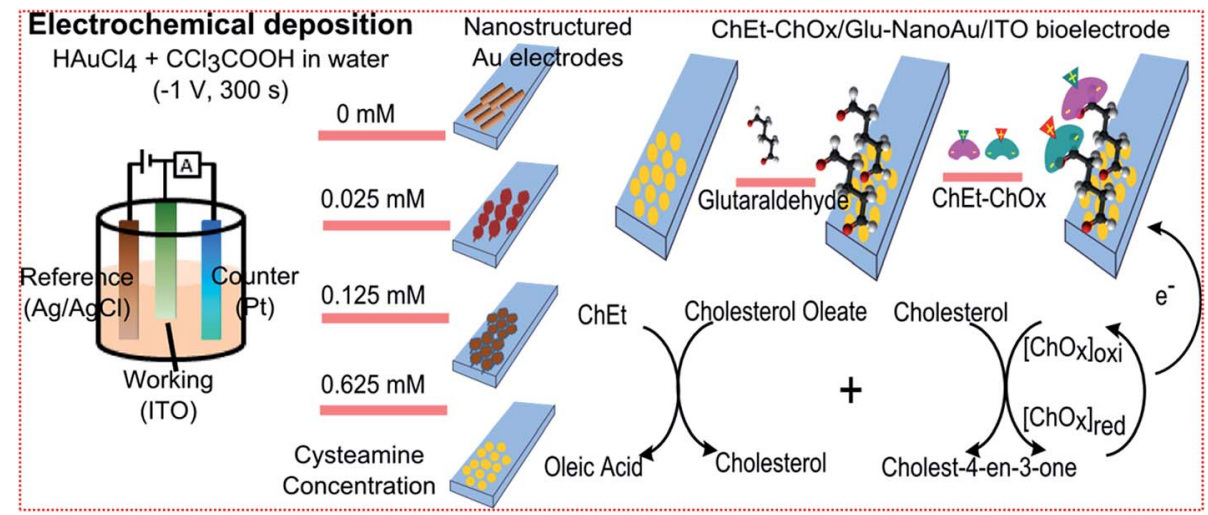

Scheme 1 Schematic illustration for the electrochemical synthesis of the Au nanostructures, the fabrication of the bi-enzymatic electrode and the detection of the cholesterol ester. 
cysteamine concentration in the electrolyte (i.e. $0.025 \mathrm{mM}$, $0.125 \mathrm{mM}$ and $0.625 \mathrm{mM}$ ) [Scheme 1].

\subsection{Fabrication of the bioelectrode}

The optimized Au electrode was utilized in the fabrication of the bi-enzymatic electrode for the detection of cholesterol oleate. The amine-functionalized nanostructured Au electrode was dipped in $0.5 \%$ glutaraldehyde solution for $4 \mathrm{~h}$, followed by rinsing with distilled water. The modified $\mathrm{Au}$ film was then incubated with $100 \mu \mathrm{L}$ of phosphate buffer solution (PBS; pH 7.4) containing ChEt and ChOx $\left(1 \mathrm{mg} \mathrm{mL}^{-1}\right)$ overnight at $4{ }^{\circ} \mathrm{C}$. The aldehyde group at the surface binds covalently with the amine group of the enzyme molecules resulting in the fabrication of the ChEt-ChOx/Glu-NanoAu/ITO bioelectrode. The loosely bound enzyme molecules were washed off with PBS buffer and the bioelectrode was stored at $4{ }^{\circ} \mathrm{C}$.
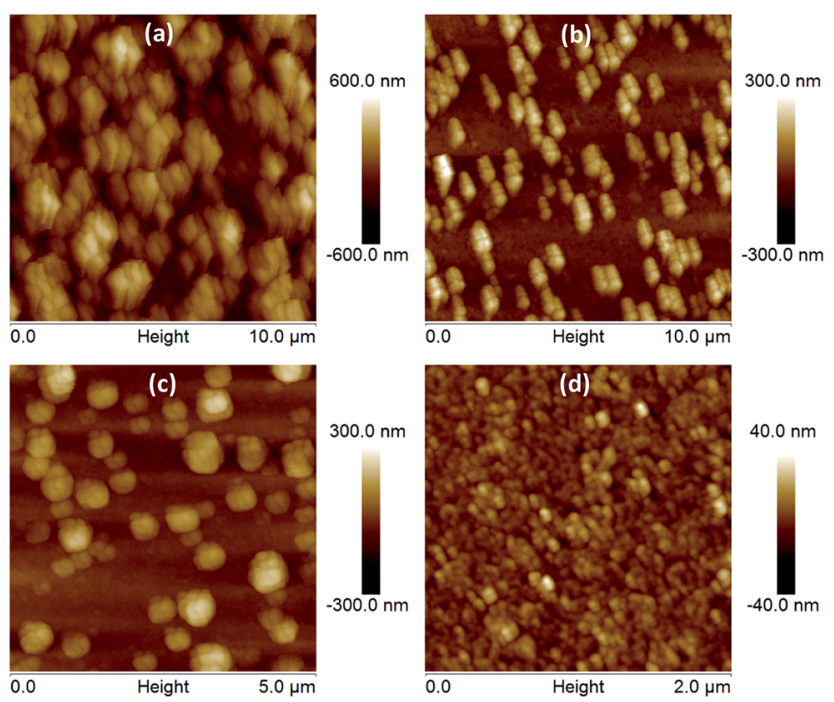

Fig. 1 AFM images of the Au films electrochemically deposited using $\mathrm{HAuCl}_{4}$ with cysteamine concentrations of (a) $0 \mathrm{mM}$, (b) $0.025 \mathrm{mM}$, (c) $0.125 \mathrm{mM}$ and (d) $0.625 \mathrm{mM}$.

\section{Results and discussion}

\subsection{AFM studies}

Fig. 1 shows the structural features of the electrochemically deposited Au nanostructures on the ITO coated glass plates. The reduced $\mathrm{HAuCl}_{4}$ molecules assemble into plate-like structures (average length $\sim 800 \mathrm{~nm}$, aspect ratio $\sim 2.5$ ) and are stacked on top of each other in the absence of capping agents [Fig. 1(a)]. On applying an electric potential, the $\mathrm{Au}^{3+}$ ions present in the electrolyte drift towards the ITO substrate where $\mathrm{Au}^{3+}$ reduces to $\mathrm{Au}^{0}$, resulting in the nucleation of $\mathrm{Au}$ NPs. The structure of the reduced $\mathrm{Au}$ is determined by the current across the electrodeelectrolyte interface. In the absence of any capping ligand, the assembly of the reduced Au into elongated structures is due to the slow rate of electrochemical reaction. On incorporation of the capping ligand (cysteamine) into the electrolyte, the agglomeration reduces due to an increase in the electrochemical current. In the presence of $0.025 \mathrm{mM}$ cysteamine, the formation of necks in the elongated rod-like structures of $\mathrm{Au}$ (average length $\sim 800 \mathrm{~nm}$, aspect ratio $\sim 2.5$ ) was observed due to the diffusion of the cysteamine molecules [Fig. 1(b)]. ${ }^{18}$ Spherical Au NPs act as building blocks for these structures and no stacking was observed. The rods fragment into circular structures of $\sim 400 \mathrm{~nm}$ with a cysteamine concentration of 0.125 $\mathrm{mM}$ [Fig. 1(c)]. On further increasing the cysteamine concentration, the coverage of cysteamine molecules over the Au NPs restricted their assembly into secondary structures and densely packed spherical Au NPs were obtained [Fig. 1(d)]. The Au NPs assembled into various structures depending on the concentration of cysteamine to attain the minimum surface energy. With sufficient cysteamine molecules, a film of densely packed $\mathrm{Au}$ NPs is obtained.

\subsection{Wettability studies of the nanostructured Au films}

Contact angle measurements were carried out to test the surface wettability of the nanocrystalline Au films [Fig. 2A]. Water was used as the dispersant and a sessile drop method was used to evaluate the contact angle of the water drop with the Au surface. It was observed that the Au film prepared in the absence of
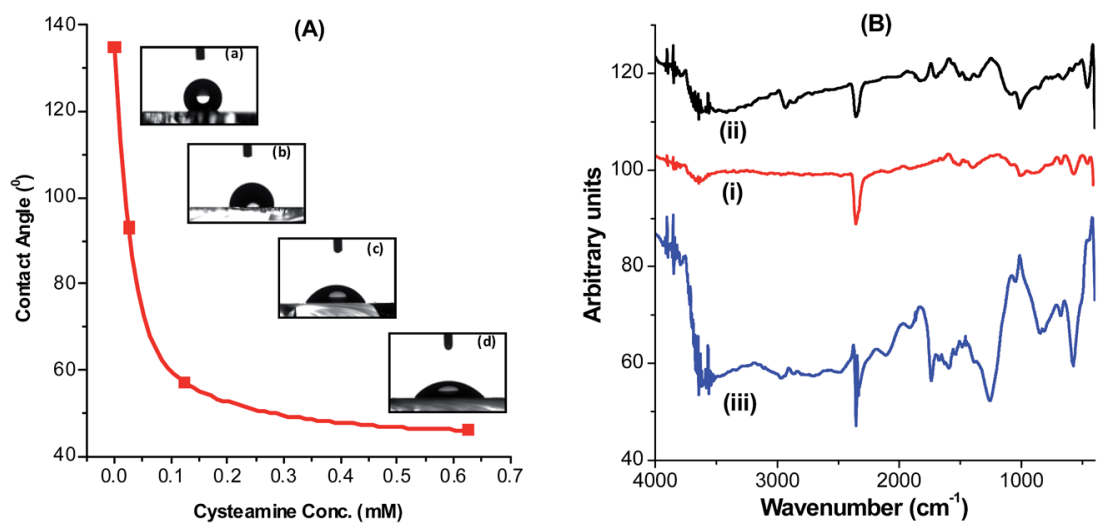

Fig. 2 (A) Contact angle of a water drop with the deposited Au films with various cysteamine concentrations of (a) $0 \mathrm{mM}$, (b) $0.025 \mathrm{mM}$, (c) 0.125 $\mathrm{mM}$ and (d) $0.625 \mathrm{mM}$. (B) FTIR spectra of the (i) NanoAu/ITO, (ii) Glu-NanoAu/ITO and (iii) ChEt-ChOx/Glu-NanoAu/ITO electrodes. 
cysteamine is highly hydrophobic with a contact angle of $135^{\circ}$ [Fig. 2A(a)] and does not allow a water drop to spread onto its surface. However, the adsorption of aqueous thiol (cysteamine) onto the Au NPs results in a decrease in the contact angle of the nanocrystalline films. When there is less cysteamine, the hydrophobic nature of the film prevails due to incomplete coverage of the NPs with cysteamine, resulting in a contact angle of $97^{\circ}$ [Fig. 2A(b)]. As the amount of cysteamine increases, more of the NP surface gets covered with the aqueous thiol making the film more hydrophilic with the contact angle to water being $57^{\circ}$ [Fig. $\left.2 \mathrm{~A}(\mathrm{c})\right]$. With an excess of cysteamine, all the surfaces of the NPs were well covered which allowed the smooth spreading of the water droplet on the surface of the film and the contact angle reduces to $46^{\circ}$ [Fig. $\left.2 \mathrm{~A}(\mathrm{~d})\right]$. This shows that cysteamine imparts hydrophilicity to the nanostructured $\mathrm{Au}$ films, which is essential for the immobilization of biomolecules. The contact angle of the water droplet on the Au surface decays exponentially with the amount of cysteamine and the evolution of the hydrophilic character of the Au films is due to the increase in amine terminations at the Au surface. ${ }^{19}$

\subsection{FTIR studies}

Fig. 2B shows the FTIR spectra of the NanoAu/ITO electrode, the Glu-NanoAu/ITO electrode and the ChEt-ChOx/Glu-NanoAu/ ITO bioelectrode. The peaks at $684 \mathrm{~cm}^{-1}, 892 \mathrm{~cm}^{-1}$ and 996 $\mathrm{cm}^{-1}$ correspond to the $\mathrm{C}-\mathrm{S}$ stretch, $\mathrm{NH}_{2}$ stretch and $\mathrm{C}-\mathrm{N}$ stretch in primary aliphatic amines, respectively, confirming the presence of cysteamine molecules in the Au film [Fig. 2B(i)]. The peaks at $449 \mathrm{~cm}^{-1}, 566 \mathrm{~cm}^{-1}, 1401 \mathrm{~cm}^{-1}$ and $1518 \mathrm{~cm}^{-1}$ arise from the alkyl group $\left(\mathrm{C}_{n} \mathrm{H}_{2 n+1}\right), \mathrm{C}-\mathrm{Cl}$ stretch, $\mathrm{C}-\mathrm{H}$ stretch and $-\mathrm{COO}^{-}$antisymmetric stretch of the trichloroacetic acid molecules. ${ }^{20}$ In Fig. $2 \mathrm{~B}(\mathrm{ii})$, the peaks at $1094 \mathrm{~cm}^{-1}, 1431 \mathrm{~cm}^{-1}$, $1507 \mathrm{~cm}^{-1}, 1692 \mathrm{~cm}^{-1}, 1814 \mathrm{~cm}^{-1}$ corresponding to the $\mathrm{C}-\mathrm{NH}_{2}$ stretch, $\mathrm{C}-\mathrm{N}$ stretch, $\mathrm{NH}_{3}{ }^{+}$deformation, $\mathrm{NH}_{2}$ stretch and $\mathrm{C}=\mathrm{O}$ stretch, respectively, confirm the formation of covalent amide bonds between the amine-terminated $\mathrm{Au}$ surface and the glutaraldehyde. The peaks at $2872 \mathrm{~cm}^{-1}$ and $2933 \mathrm{~cm}^{-1}$ correspond to the $\mathrm{C}-\mathrm{H}$ antisymmetric and symmetric stretches. Additionally, in Fig. 2B(iii), the peaks at $826 \mathrm{~cm}^{-1}, 1048 \mathrm{~cm}^{-1}$ and $2335 \mathrm{~cm}^{-1}$ corresponding to the $\mathrm{P}-\mathrm{O}$ stretch, $\mathrm{P}-\mathrm{OH}$ stretch and $\mathrm{P}-\mathrm{H}$ stretch, respectively, arise from the enzyme molecules. ${ }^{20}$ The peaks at $1921 \mathrm{~cm}^{-1}$ and $2106 \mathrm{~cm}^{-1}$ arise from the combination of $\mathrm{NH}_{3}{ }^{+}$torsion and $\mathrm{NH}_{3}{ }^{+}$antisymmetric deformation and the peaks at $1592 \mathrm{~cm}^{-1}$ and $1723 \mathrm{~cm}^{-1}$ arise from the $\mathrm{NH}_{2}$ stretch and $\mathrm{C}=\mathrm{O}$ stretch, respectively, due to the amide bond formation between glutaraldehyde and the enzyme molecules. ${ }^{20}$ This confirms the covalent attachment of ChEtChOx molecules to the nanostructured Au surface.

\subsection{Morphological characterization of the bioelectrode}

Morphological studies were carried out to investigate the loading of enzyme molecules onto the nanostructured $\mathrm{Au}$ electrode and the SEM images are displayed in Fig. 3. The micrograph [Fig. 3(a)] reveals a dense packing of the uniformsized Au NPs onto the ITO surface, in agreement with the AFM image. The morphology of the nanostructured Au electrode changes to spherical globules on incubation with glutaraldehyde [Fig. 3(b)]. Moreover, the morphology changes from globular spheres of glutaraldehyde to densely packed agglomerates on incubation with the enzyme solution [Fig. 3(c)]. This confirms the adsorption of enzyme molecules onto the nanostructured $\mathrm{Au}$ electrode, leading to the formation of the bioelectrode.

\subsection{Electrochemical characterization of the nanostructured Au films}

Fig. 4(A) shows the current response as a function of time during electrochemical deposition of the $\mathrm{Au}$ nanostructures. The current across the electrode-electrolyte system was $\sim 10 \mathrm{~mA}$ in the absence of cysteamine and elongated plate-like structures result. On incorporation of cysteamine, the current was found to increase. This results in a higher drift of the $\mathrm{HAuCl}_{4}$ molecules towards the electrode and thus faster electroreduction of $\mathrm{Au}^{3+}$ ions. The subsequent addition of more cysteamine to the electrolyte solution leads to an increase in current to $\sim 20 \mathrm{~mA}$. The amount of cysteamine and the rate of electroreduction of $\mathrm{Au}^{3+}$ ions govern the assembly of reduced $\mathrm{Au}$ on the ITO surface. The Au NPs assemble into different nanostructures in order to attain minimum surface energy and so a higher amount of cysteamine results in the deposition of smaller nanostructures.

Cyclic voltammetric studies were carried out to examine the electrochemical behaviour of the nanostructured $\mathrm{Au}$ films in PBS without any redox mediator [Fig. 4(B)]. The nanostructured Au films exhibited redox peaks at $\sim 0.2 \mathrm{~V}$ and $\sim 0.0 \mathrm{~V}$, which are

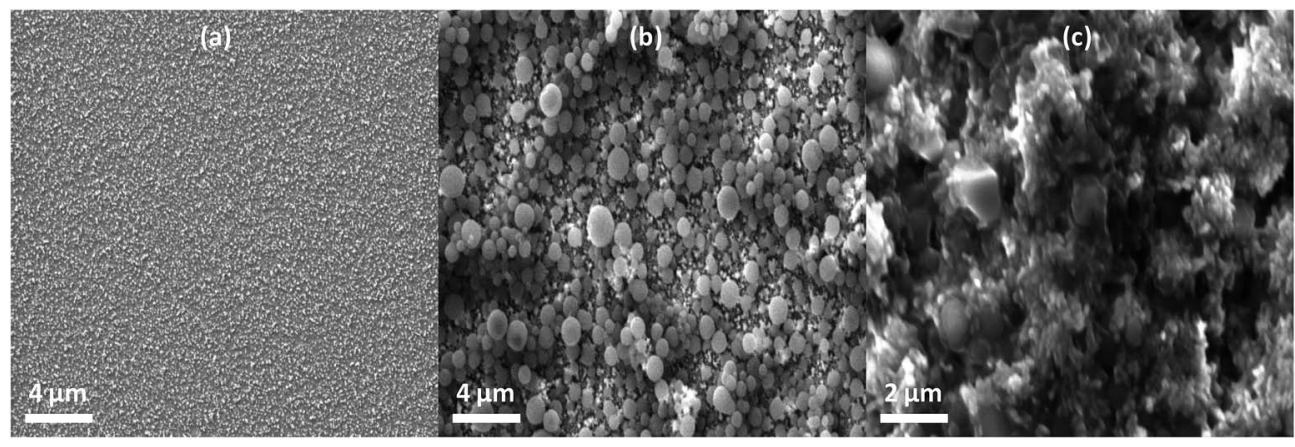

Fig. 3 SEM images of the (a) NanoAu/ITO, (b) Glu-NanoAu/ITO and (c) ChEt-ChOx/Glu-NanoAu/ITO electrodes. 

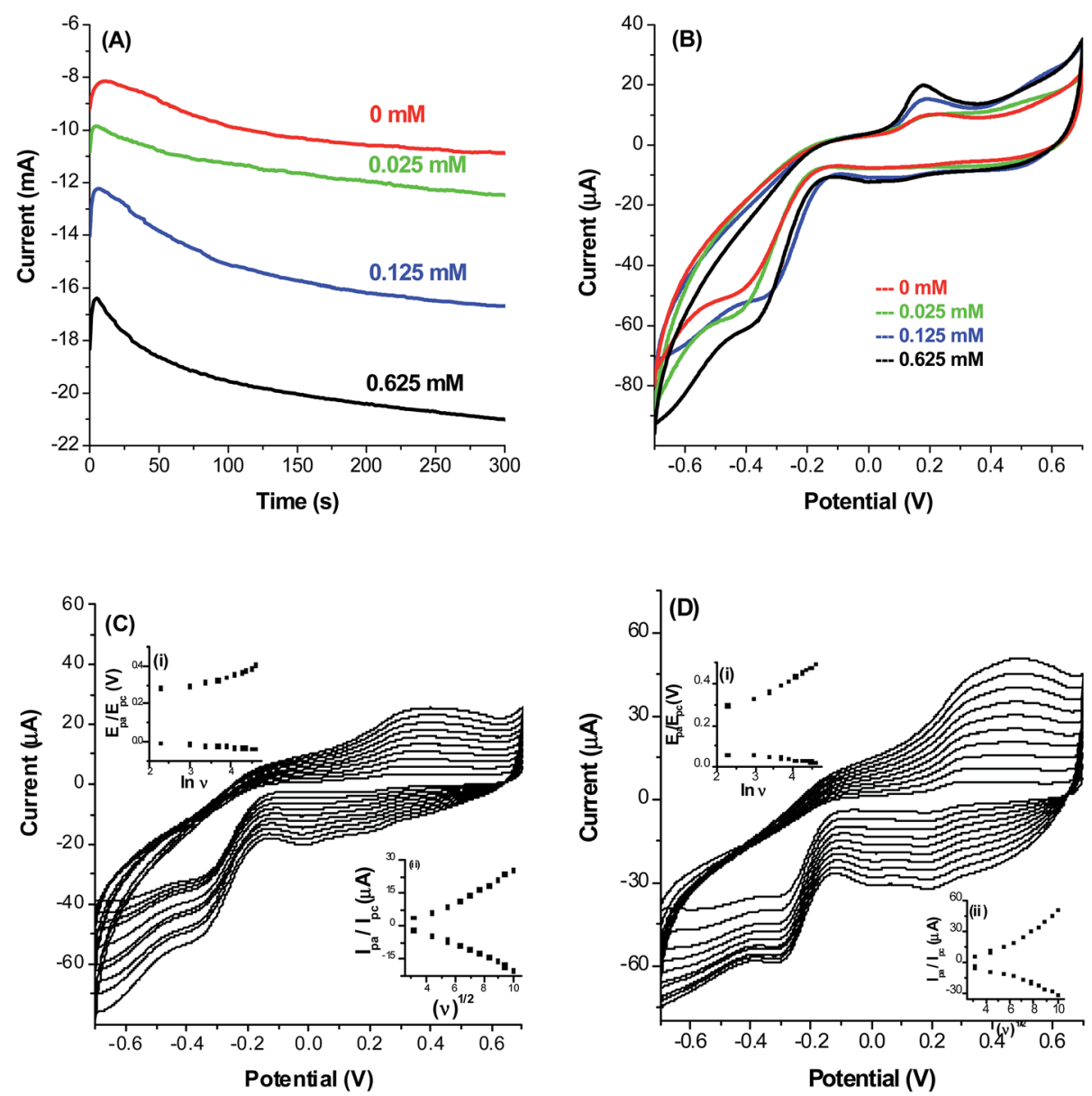

Fig. 4 (A) Chronoamperometric response as a function of time during electrochemical deposition of the Au nanostructures. (B) Cyclic voltammograms of the nanostructured Au electrodes in phosphate buffer saline $(\mathrm{pH} 7.4)$ without mediator at a scan rate of $50 \mathrm{mV} \mathrm{s}^{-1}$. Cyclic voltammograms as a function of scan rate for (C) the NanoAu/ITO electrode and (D) the ChEt-ChOx/Glu-NanoAu/ITO bioelectrode in phosphate buffer saline-(pH 7.4). Inset (i) the anodic and cathodic potentials as a function of the logarithm of the scan rate. Inset (ii) the anodic and cathodic currents as a function of the square root of the scan rate.

attributed to the oxidation and subsequent reduction of gold oxide (AuOx) as a result of the electronic polarization. ${ }^{21}$ However, the difference in magnitudes of the anodic and cathodic current indicates the chemical irreversibility of the surface reaction. With the incorporation of cysteamine, the sulphur ions at the surface of the Au nanostructures further facilitate the electrochemical oxidation-reduction of $\mathrm{Au}$, resulting in an enhanced electrochemical current. ${ }^{22}$ Also, the higher surface area provided by the smaller Au nanostructures results in faster electron transfer kinetics leading to an increase in the redox current.

\subsection{Kinetic studies of the Au electrode and the bioelectrode}

Cyclic voltammetric studies for the NanoAu/ITO electrode and the ChEt-ChOx/Glu-NanoAu/ITO bioelectrode as a function of scan rate $\left(10-100 \mathrm{mVs}^{-1}\right)$ were carried out to investigate the interfacial phenomena [Fig. 4C and D]. The shift in the anodic potential towards a positive value as scan rate increases implies that the electrochemical reaction is either quasi-reversible or irreversible in nature. As the anodic and cathodic currents vary linearly with the square root of the scan rate (above $30 \mathrm{mV} \mathrm{s}^{-1}$ ), this suggests that the electrochemical reaction is quasireversible at low scan rates $\left(<30 \mathrm{mV} \mathrm{s}^{-1}\right)$ and irreversible above $30 \mathrm{mV} \mathrm{s}^{-1}$.

Furthermore, the anodic peak potential varies linearly as a logarithmic function of the scan rate in the range $10-100 \mathrm{mV} \mathrm{s}^{-1}$ [Fig. 4C and D (inset (i))]. Although the shift in the cathodic potential is not appreciable, a slight shift can be seen upon magnifying the curves. The value of the electron transfer coefficient $(\alpha)$ for $n$ number of electrons was determined from the anodic and cathodic slopes using Laviron's equations: $2.303 R T /$ $(1-\alpha) n F$ and $-2.303 R T / \alpha n F$, respectively. ${ }^{23}$ The value of $\alpha$ for the $\mathrm{Au}$ electrodes increases from 0.50 to 0.65 as the cysteamine concentration increases [Table 1]. The charge transfer rate constant $\left(k_{\mathrm{s}}\right)$ was calculated using eqn (1):

$$
\begin{aligned}
\ln k_{\mathrm{s}}= & \alpha \ln (1-\alpha)+(1-\alpha) \ln \alpha-\ln \left(\frac{R T}{n F v}\right) \\
& -\alpha(1-\alpha)\left(\frac{n F \Delta E_{\mathrm{p}}}{R T}\right)
\end{aligned}
$$


Table 1 The kinetic parameters for the various nanostructured Au electrodes and the bioelectrode

\begin{tabular}{|c|c|c|c|c|}
\hline Electrodes & $\begin{array}{l}\text { Cysteamine } \\
\text { conc. }(\mathrm{mM})\end{array}$ & $\begin{array}{l}\text { Electron transfer } \\
\text { coefficient, } \alpha\end{array}$ & $\begin{array}{l}\text { Charge transfer } \\
\text { rate constant, } k_{\mathrm{s}}\left(\mathrm{s}^{-1}\right)\end{array}$ & $\begin{array}{l}\text { Surface conc., } \\
\Gamma\left(\mathrm{mol} \mathrm{cm}^{-2}\right)\end{array}$ \\
\hline NanoAu/ITO & 0 & 0.50 & 68.39 & $1.30 \times 10^{-13}$ \\
\hline NanoAu/ITO & 0.125 & 0.62 & 9.78 & $3.72 \times 10^{-13}$ \\
\hline NanoAu/ITO & 0.625 & 0.65 & 3.55 & $8.22 \times 10^{-13}$ \\
\hline ChEt-ChOx/Glu-NanoAu/ITO & 0.625 & 0.68 & 7.09 & $8.82 \times 10^{-12}$ \\
\hline
\end{tabular}

The value of $k_{\mathrm{s}}$ for the $\mathrm{Au}$ electrodes decreases from 68.39 to $3.55 \mathrm{~s}^{-1}$ as the cysteamine concentration increases [Table 1]. This is due to the slight increase in the peak separation of the Au electrodes as the cysteamine concentration increases.

For a surface-controlled process, the surface concentration of the ionic species $(T)$ can be estimated using the BrownAnson model [eqn (2)]: ${ }^{24}$

$$
I_{\mathrm{p}}=n^{2} F^{2} v A \Gamma(4 R T)^{-1}
$$

where $I_{\mathrm{p}} / \nu$ can be obtained from the linear regression of the anodic peak potential versus the scan rate curve. The surface concentration of the ionic species on the Au electrodes increases with increasing cysteamine concentration from 1.30 $\times 10^{-13}$ to $8.22 \times 10^{-12} \mathrm{~mol} \mathrm{~cm}{ }^{-2}$. This is in accordance with the higher loading of gold nanostructures on the ITO substrate with the increase in cysteamine concentration. The nanostructured Au electrode ( $0.625 \mathrm{mM}$ cysteamine) with the highest surface concentration of the electroactive species was used for the fabrication of the enzymatic bioelectrode.
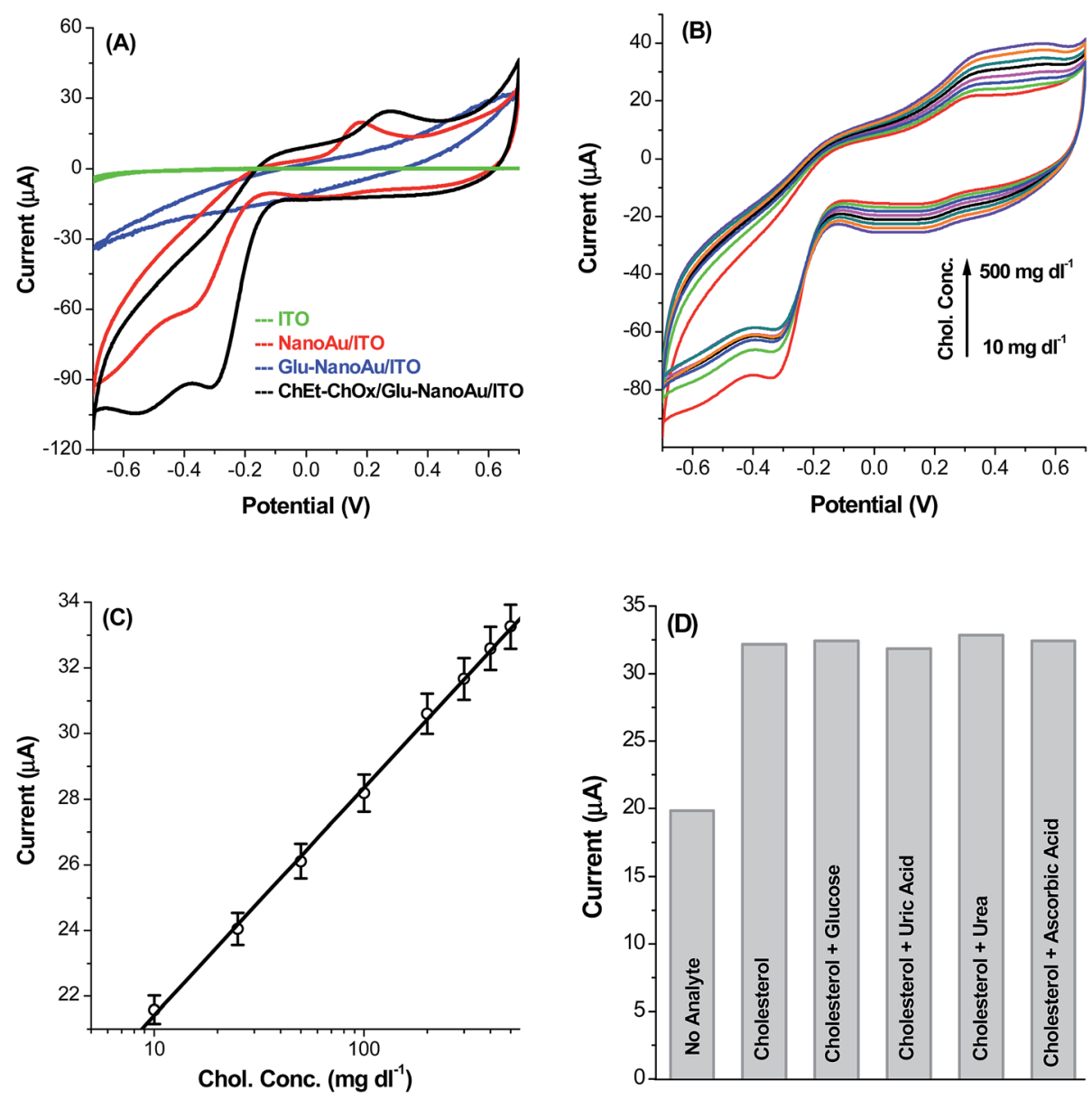

Fig. 5 (A) Cyclic voltammograms of the ITO electrode, NanoAu/ITO electrode, Glu-NanoAu/ITO electrode and ChEt-ChOx/Glu-NanoAu/ITO bioelectrode in phosphate buffer saline at a scan rate of $50 \mathrm{mV} \mathrm{s}^{-1}$. (B) Cyclic voltammograms of the ChEt-ChOx/Glu-NanoAu/ITO bioelectrode in the presence of differing cholesterol concentrations (10-500 $\mathrm{mg} \mathrm{dl}^{-1}$ ) in phosphate buffer saline (pH 7.4). (C) Calibration plot showing the variation of anodic current with differing cholesterol concentrations. (D) Interference curve showing the variation of anodic current as a function of different analytes. 
After ChEt-ChOx immobilization, the value of $k_{\mathrm{s}}$ increases from 3.55 to $7.09 \mathrm{~s}^{-1}$. This is attributed to the establishment of electrical contact between the redox centres of the enzyme molecules and the surface of the $\mathrm{Au}$ nanostructures which facilitates electronic and ionic transport across the interface. Also, the surface concentration of the ionic species was found to increase ten-fold from $8.22 \times 10^{-13}$ to $8.82 \times 10^{-12} \mathrm{~mol} \mathrm{~cm}^{-2}$ after enzyme immobilization [Table 1]. This reveals the chemisorption of enzyme molecules onto the nanostructured $\mathrm{Au}$ electrode, resulting in a greater number of active sites on the nanostructured Au matrix. The ChEt-ChOx/Glu-NanoAu/ITO bioelectrode fabricated using the optimized $\mathrm{Au}$ electrode (0.625 $\mathrm{mM}$ cysteamine) was utilized for the electrochemical detection of cholesterol oleate.

\subsection{Cyclic voltammetric response of the bioelectrode}

The fabrication of the ChEt-ChOx/Glu-NanoAu/ITO bioelectrode was investigated at each modification step using cyclic voltammetry in PBS [Fig. 5A]. The ITO electrode has no current response in the potential region from $-0.7 \mathrm{~V}$ to $+0.7 \mathrm{~V}$. The redox peaks from the $\mathrm{Au}$ nanostructures at $\sim 0.2 \mathrm{~V}$ and $\sim 0.0 \mathrm{~V}$ disappear on incubation of the Au film with glutaraldehyde and a remarkable decrease in current was observed. The insulating layer of glutaraldehyde onto the nanostructured Au electrode results in sluggish electron transport across the interface. After ChEt-ChOx immobilization, the redox peaks reappear at $\sim 0.3 \mathrm{~V}$ and $\sim 0.0 \mathrm{~V}$, which are attributed to the electronic communication of the flavin adenine dinucleotide (FAD) cofactor of the enzyme molecules with the $\mathrm{Au}$ nanostructures. ${ }^{25}$ The electrical wiring of the FAD molecules and Au nanostructures results in direct electron transfer and thus paves the way for the fabrication of a mediator-free total cholesterol biosensor.

The electrochemical response of the ChEt-ChOx/GluNanoAu/ITO bioelectrode towards different concentrations of cholesterol ester (oleate) was studied [Fig. 5B]. The anodic current was found to increase with increasing concentrations of cholesterol ester (10-500 $\left.\mathrm{mg} \mathrm{dl}^{-1}\right)$. In the proposed biochemical reaction, the cholesterol ester is hydrolyzed via cholesterol esterase to cholesterol (or $3 \beta$-hydroxysteroid) and fatty acid. ChOx then catalyzes the oxidation of cholesterol (3 $\beta$-hydroxysteroid) to the intermediate product $\Delta 5$-6-ene-3 $\beta$-ketosteroid (cholest-5-en-3-one). The isomerization of this intermediate product results in $\Delta 3$-4-ene-3 $\beta$-ketosteroid (cholest-4-en-3one). ${ }^{26}$ The FAD cofactor gets reduced to $\mathrm{FADH}_{2}$ during this process and in order to re-oxidize, the direct exchange of electrons with the nanostructured Au electrode occurs, resulting in an increase in peak current as the concentration of cholesterol oleate increases. The anodic peak current of the ChEt-ChOx/Glu-NanoAu/ITO bioelectrode plotted as a logarithmic function of the cholesterol concentration reveals a linearity range of $10-500 \mathrm{mg} \mathrm{dl}^{-1}$ (within $2 \%$ error) and a standard deviation and correlation coefficient of $0.28 \mu \mathrm{A}$ and 0.99, respectively [Fig. 5C]. The sensitivity of the ChEt-ChOx/ Glu-NanoAu/ITO bioelectrode, calculated from the slope of the linear regression curve, was $0.53 \mathrm{~mA} \mathrm{mM} \mathrm{mm}^{-1}$ and the detection limit calculated using $3 * \mathrm{SD} /$ sensitivity was $1.57 \mu \mathrm{M}$. The apparent Michaelis-Menten constant, which is an indication of the enzyme-substrate kinetics, was evaluated from the Lineweaver-Burk equation and was calculated to be $0.63 \mathrm{mM} .^{27}$ The small $K_{\mathrm{m}}^{\mathrm{app}}$ value indicates that the immobilized enzymes possess high enzymatic activity and that the fabricated biosensor exhibits a high affinity for cholesterol. Table 2 summarizes the biosensing characteristics of the mediator-free cholesterol biosensors reported in literature. It can be seen that the sensitivity of the proposed total cholesterol biosensor is much higher and the limit of detection is appreciably lower as compared to those reported in the literature. Also, the proposed biosensor has a comparable value of $K_{\mathrm{m}}^{\mathrm{app}}$ and an extended linear range for esterified cholesterol detection.

The effect of potential interferents on the total cholesterol measurements was investigated by taking the solution containing a $1: 1$ ratio of cholesterol ester $\left(200 \mathrm{mg} \mathrm{dl}^{-1}\right)$ and interferents such as glucose $(5 \mathrm{mM})$, urea $(1 \mathrm{mM})$, uric acid (0.1 $\mathrm{mM})$ and ascorbic acid (0.05 mM) [Fig. 5D]. In the presence of interferents, a $2-3 \%$ change in current was observed from the $\mathrm{CV}$ response of the ChEt-ChOx/Glu-NanoAu/ITO bioelectrode. This was calculated using the equation: \%inter $=I_{\text {chol }}-I_{\text {Int }} /$ $I_{\text {chol}}$, where $I_{\text {chol }}$ and $I_{\text {int }}$ are the changes in current corresponding to the cholesterol ester and the $1: 1$ mixture of the cholesterol ester with the interferent, respectively. This shows that the proposed biosensor is highly selective towards total cholesterol.

\subsection{Clinical sample analysis}

The response studies of the ChEt-ChOx/Glu-NanoAu/ITO bioelectrode were carried out using five clinical samples with varying cholesterol concentrations. Serum samples from patients along with clinical data of the cholesterol levels were collected from Dr Arvind's Family Clinic, New Delhi (India). In comparison to the standard cholesterol concentration, it was observed that the

Table 2 Comparison table summarizing the biosensing characteristics of mediator-free cholesterol biosensors

\begin{tabular}{|c|c|c|c|c|c|c|}
\hline ChOx/AuNPs-MWCNT/GCE & Free cholesterol & - & $4.3 \mu \mathrm{M}$ & $0.01-5 \mathrm{mM}$ & $0.29 \mathrm{mM}$ & 28 \\
\hline $\mathrm{ChOx} / \mathrm{Au}-\mathrm{ODA} / \mathrm{ITO}$ & Free cholesterol & $1.08 \times 10^{-3} \mathrm{~mA} \mathrm{mM}^{-1}$ & $0.60 \mathrm{mM}$ & $0.65-12.95 \mathrm{mM}$ & $0.5 \mathrm{mM}$ & 29 \\
\hline ChOx-Glu/Cys-Au/ITO & Free cholesterol & $4.22 \mathrm{~mA} \mathrm{mM}{ }^{-1} \mathrm{~cm}^{-2}$ & $5.41 \mu \mathrm{M}$ & $0.26-12.95 \mathrm{mM}$ & $0.57 \mathrm{mM}$ & 16 \\
\hline ChEt-ChOx/4-ATP/Au & Total cholesterol & $886.6 \mathrm{nA} \mathrm{mM}^{-1}$ & - & $0.62-10 \mathrm{mM}$ & $1.06 \mathrm{mM}$ & 30 \\
\hline ChEt-ChOx/Glu-NanoAu/ITO & Total cholesterol & $0.53 \mathrm{~mA} \mathrm{mM}^{-1} \mathrm{~cm}^{-2}$ & $1.57 \mu \mathrm{M}$ & $0.26-12.95 \mathrm{mM}$ & $0.63 \mathrm{mM}$ & $\begin{array}{l}\text { Present } \\
\text { work }\end{array}$ \\
\hline
\end{tabular}



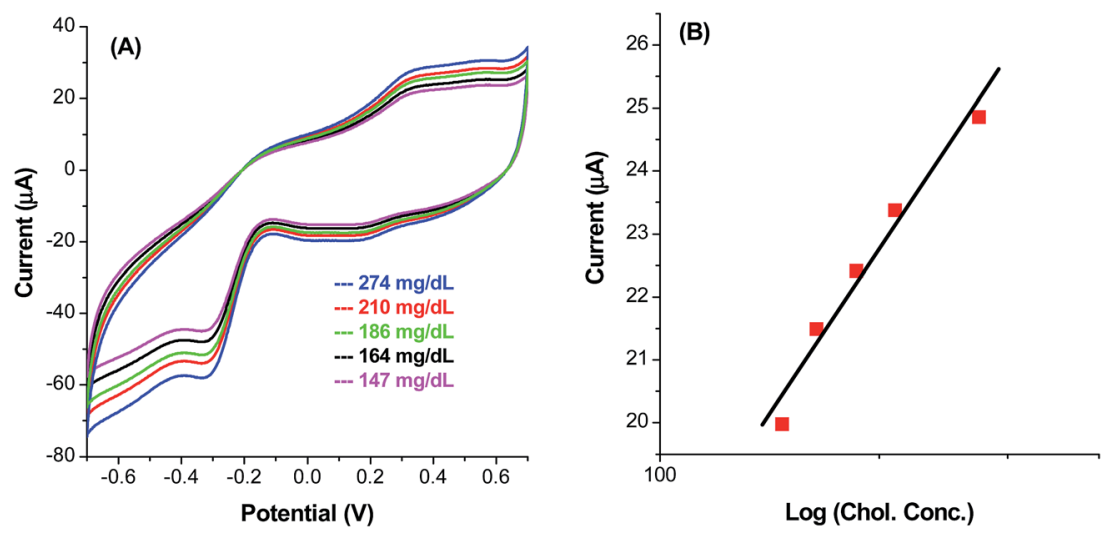

Fig. 6 (A) Cyclic voltammetric response of the ChEt-ChOx/Glu-NanoAu/ITO bioelectrode for available clinical samples of different concentrations. (B) Calibration plot showing the variation of anodic peak current with cholesterol concentrations.
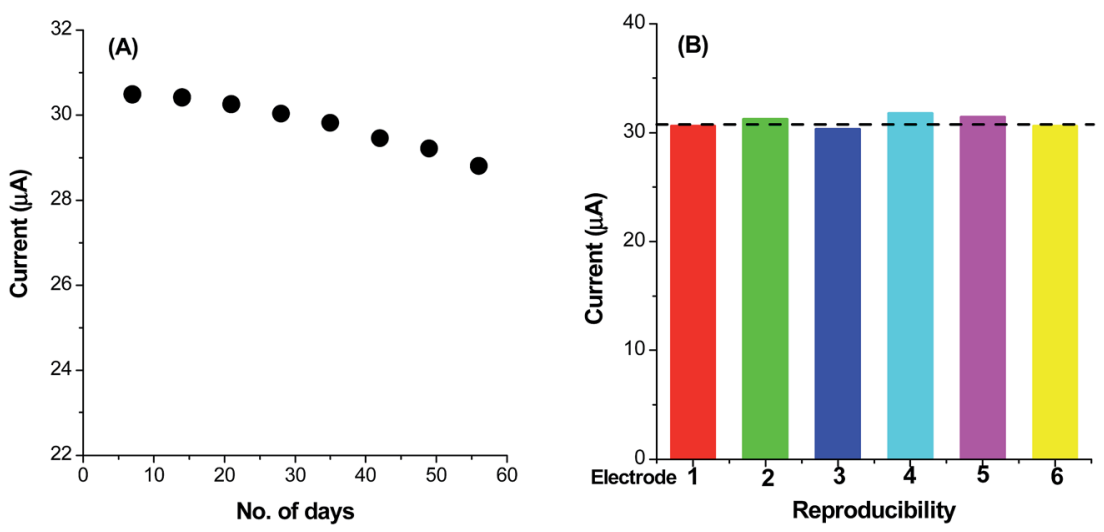

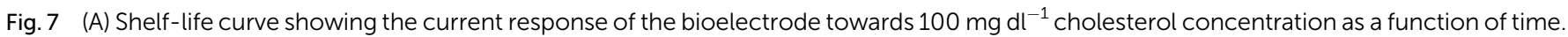
(B) Reproducibility curve showing the current response of six bioelectrodes (prepared under similar conditions) towards $100 \mathrm{mg} \mathrm{dl}^{-1} \mathrm{cholesterol}^{-1}$ concentration.

ChEt-ChOx/Glu-NanoAu/ITO bioelectrode showed a minute difference of $3-5 \%$ in the current response during cholesterol detection using the clinical serum samples [Fig. 6A]. The plot of current versus logarithmic value of concentration shows a near linear response with clinical patient samples [Fig. 6B] and the sensitivity $\left(0.48 \mathrm{~mA} \mathrm{mM}^{-1}\right)$ differs to the sensitivity for standard cholesterol concentrations by $\sim 10-11 \%$, which is acceptable. This indicates great potential of the bioelectrode for the development of point of care diagnostics. Thus, these results indicate that this novel biosensor has the potential to directly detect cholesterol in human serum samples.

The shelf life and reproducibility of the ChEt-ChOx/GluNanoAu/ITO bioelectrode were investigated using cyclic voltammetry. The bioelectrode exhibits only a $6 \%$ reduction in peak current after 8 weeks for $100 \mathrm{mg} \mathrm{dl}{ }^{-1}$ cholesterol concentration when stored at $4{ }^{\circ} \mathrm{C}$ indicating good stability [Fig. 7A]. To check the reproducibility of the proposed biosensor, six bioelectrodes were prepared under similar conditions and their current responses were studied in the presence of $100 \mathrm{mg} \mathrm{dl} \mathrm{di}^{-1}$ cholesterol concentration. The biosensor showed excellent reproducibility as evidenced by the low RSD of $1.85 \%$ for the six different electrodes [Fig. 7B].

\section{Conclusions}

A one-step method for the fabrication of functionalized nanostructured Au electrodes via an electrochemical route has been demonstrated. The morphology and the hydrophilicity of the nanostructured Au electrode were controlled using cysteamine. The variation of cysteamine concentration during fabrication enhances the electrochemical activity of the nanostructured $\mathrm{Au}$ electrode. The cysteamine molecules provide $\mathrm{NH}_{2}$ terminations at the Au surface resulting in covalent functionalization of the bi-enzymes (ChEt-ChOx). The electrochemically deposited Au nanostructures on the ITO substrates exhibit excellent electrocatalytic activity and en route direct transfer of electrons from the immobilized enzyme molecules to the nanostructured electrode resulting in a mediator-free $3^{\text {rd }}$-generation total cholesterol biosensor. The fabricated bioelectrode was investigated for its kinetic parameters and its biosensing performance. The optimized bioelectrode exhibits a high sensitivity of 0.53 $\mathrm{mA} \mathrm{mM} \mathrm{mm}^{-1} \mathrm{~cm}^{-2}$, a low detection limit of $1.57 \mu \mathrm{M}$ and a low $K_{\mathrm{m}}^{\mathrm{app}}$ value of $0.63 \mathrm{mM}$ due to the facile and direct charge transportation across the interface. The proposed biosensor shows good selectivity, reproducibility and stability. 


\section{Acknowledgements}

We thank Prof. R. C. Budhani (Director, NPL), Dr A. M. Biradar and Prof. B. D. Malhotra for providing support and facilities, and Dr K. N. Sood for SEM measurements. R. S. is thankful to UGC-CSIR for the award of a Junior Research Fellowship. Financial support received from DST and CSIR Empower projects is sincerely acknowledged.

\section{References}

1 T. K. Sau, A. L. Rogach, F. Jackel, T. A. Klar and J. Feldmann, Adv. Mater., 2010, 22, 1805.

2 F. C. Chen, J. L. Wu, C. L. Lee, Y. Hong, C. H. Kuo and M. H. Huang, Appl. Phys. Lett., 2009, 95, 013305.

3 M. E. Stewart, C. R. Anderton, L. B. Thompson, J. Maria, S. K. Gray, J. A. Rogers and R. G. Nuzzo, Chem. Rev., 2008, 108, 494.

4 B. D. Chithrani, A. A. Ghazani and W. C. W. Chan, Nano Lett., 2006, 6, 662.

5 P. K. Jain, K. S. Lee, I. H. El Sayed and M. A. El Sayed, J. Phys. Chem. B, 2006, 110, 7238.

$6 \mathrm{M} . \mathrm{Hu}$, J. Chen, Z. Y. Li, L. Au, G. V. Hartland, X. Li, M. Marquez and Y. Xia, Chem. Soc. Rev., 2006, 35, 1084.

7 B. Duncan, C. Kim and V. M. Rotello, J. Controlled Release, 2010, 148, 122.

8 C. M. Cobley, J. Chen, E. C. Cho, L. V. Wang and Y. Xia, Chem. Soc. Rev., 2011, 40, 44.

9 P. Si, P. Kannan, L. Guo, H. Son and D. H. Kim, Biosens. Bioelectron., 2011, 26, 3845.

10 M. C. Daniel and D. Astruc, Chem. Rev., 2003, 104, 293.

11 L. Shi, Z. Chu, Y. Liu, W. Jin and X. Chen, Biosens. Bioelectron., 2013, 49, 184.

12 H. Liu, Y. Tian and P. Xia, Langmuir, 2008, 24, 6359.

13 I. Zhitomirsky, J. Mater. Sci., 2006, 41, 8186.
14 C. J. Huang, P. H. Chiu, Y. H. Wang, K. L. Chen, J. J. Linn and C. F. Yang, J. Electrochem. Soc., 2006, 153, D193.

15 L. Wang, X. Chen, X. Wang, X. Han, S. Liu and C. Zhao, Biosens. Bioelectron., 2011, 30, 151.

16 R. Sharma, M. A. Ali, N. R. Selvi, V. N. Singh, R. K. Sinha and V. V. Agrawal, J. Phys. Chem. C, 2014, 118, 6261.

17 Z. Luo, K. Zheng and J. Xie, Chem. Commun., 2014, 50, 5143.

18 H. G. Liao, L. Cui, S. Whitelam and H. Zheng, Science, 2012, 336, 1011.

19 A. F. Azevedo, J. T. Matsushima, F. C. Vicentin, M. R. Baldan and N. G. Ferreira, Appl. Surf. Sci., 2009, 255, 6565.

20 R. K. Dukor, J. M. Chalmers and P. R. Griffiths, Vibrational Spectroscopy in the Detection of Cancer, John Wiley and Sons, New York, 2001, p. 3335.

21 U. Koelle and A. Laguna, Inorg. Chim. Acta, 1999, 290, 44.

22 P. Lessner, J. Winnick, F. R. McLarnon and E. J. Cairns, J. Electrochem. Soc., 1986, 133, 2517.

23 E. Laviron, J. Electroanal. Chem. Interfacial Electrochem., 1979, 100, 263.

24 E. Laviron, J. Electroanal. Chem. Interfacial Electrochem., 1979, 101, 19.

25 S. Bhattacharyya, M. T. Stankovich, D. G. Truhlar and J. Gao, J. Phys. Chem. A, 2007, 111, 5729.

26 M. Yamashita, M. Toyama, H. Ono, I. Fujii, N. Hirayama and Y. Murooka, Protein Eng., 1998, 11, 1075.

27 A. Ahmadalinezhad and A. Chen, Biosens. Bioelectron., 2011, 26, 4508.

28 L. Zhu, L. Xu, L. Tan, H. Tan, S. Yang and S. Yao, Talanta, 2012, 106, 192.

29 Z. Matharu, P. Pandey, M. K. Pandey, V. Gupta and B. D. Malhotra, Electroanalysis, 2009, 21, 1587.

30 Z. Matharu, P. R. Solanki, V. Gupta and B. D. Malhotra, Analyst, 2012, 137, 747. 